\title{
Performance measurements at the SLS SIM beamline
}

\author{
U. Flechsig, F. Nolting, A. Fraile Rodríguez, J. Krempaský, C. Quitmann, \\ T. Schmidt, S. Spielmann and D. Zimoch
}

Paul Scherrer Institut, Swiss Light Source, 5232 Villigen PSI, Switzerland

\begin{abstract}
The Surface/Interface: Microscopy beamline of the Swiss Light Source started operation in 2001. In 2007 the beamline has been significantly upgraded with a second refocusing section and a blazed grating optimized for high photon flux. Two Apple II type undulators with a plane grating monochromator using the collimated light scheme deliver photons with an energy from $90 \mathrm{eV}$ to about $2 \mathrm{keV}$ with variable polarization for the photoemission electron microscope (PEEM) as the primary user station. We measured a focus of $(45 \times 60) \mu \mathrm{m}(v \times h)$ and a photon flux $>10^{12}$ photon/s for all gratings. Polarization switching within a few seconds is realized with the small bandpass of the monochromator and a slight detuning of the undulator.
\end{abstract}

Keywords: X-rays, microscopy, beamline optics, helicity switching, synchrotron radiation PACS: 07.85.Qe, 42.79.Dj

\section{INTRODUCTION}

The Surface/Interface: Microscopy (SIM) beamline belongs to the first four beamlines at the Swiss Light Source (SLS) which started operation in 2001. Since then the beamline has proven to be a very successful and reliable instrument for the users. Two $3.8 \mathrm{~m}$ long Apple II type undulators in the X11M straight section of SLS followed by a plane grating monochromator (PGM) using the collimated light scheme [1] provide the light for the photoemission electron microscope (PEEM). The beamline has been significantly upgraded in 2007. We installed an additional grating and a retractable second refocusing section downstream the PEEM to allow the operation of user- brought stations with a high flux density. The beamline covers the photon energy range from $90 \mathrm{eV}$ to about $2 \mathrm{keV}$ with variable polarization. With the PGM scheme the user can trade resolving power, photon flux and higher order contamination by varying the fix focus constant $c_{f f}$. The two undulators can be operated independently or with an electromagnetic chicane in phase matched mode as one long device. The features and performance of the beamline are similar to its sister beamline the Surface/Interface: Spectroscopy (SIS) beamline [2,3] which covers the lower part of the spectrum down to $9 \mathrm{eV}$.

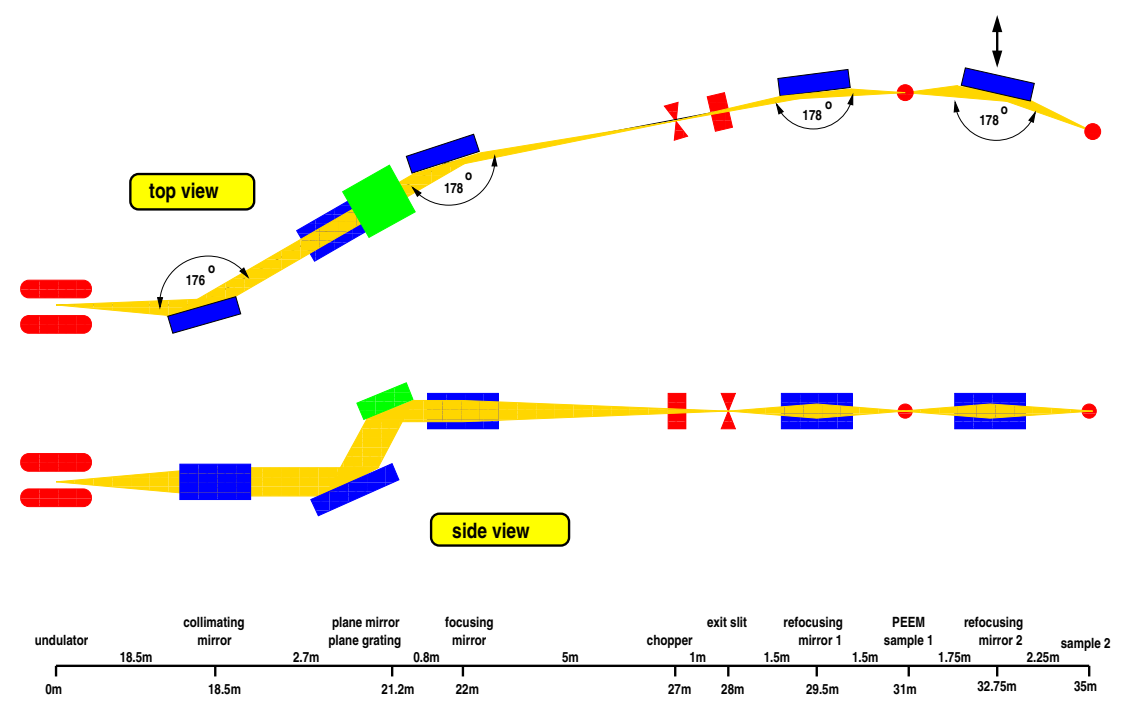

FIGURE 1. Optical layout of the SIM beamline. 
TABLE 1. Selected data of the optical elements.

\begin{tabular}{lrrrrrrrr}
\hline parameter & CM & PM & G1 & G2 & G3 & FM & RM1 & RM2 \\
\hline shape & cylinder & flat & flat & flat & flat & toroid & toroid & toroid \\
position $(\mathrm{m})$ & 18.5 & $\sim 21$ & 21.2 & 21.2 & 21.2 & 22 & 29.5 & 32.75 \\
max. power $(\mathrm{W})$ & 630 & 120 & 4 & 4 & 3 & 1.5 & - & - \\
max. pow. dens. $\left(\mathrm{mW} / \mathrm{mm}^{2}\right)$ & 620 & 280 & 2 & 3 & 3 & 0.4 & - & - \\
max. $4 \sigma$ footprint $\left(\mathrm{mm}^{2}\right)$ & $156 \times 4$ & $216 \times 3$ & $120 \times 4$ & $120 \times 4$ & $120 \times 4$ & $360 \times 25$ & $180 \times 8$ & $200 \times 8$ \\
geom. surface size $\left(\mathrm{mm}^{2}\right)$ & $270 \times 40$ & $310 \times 50$ & $100 \times 20$ & $100 \times 20$ & $100 \times 20$ & $250 \times 40$ & $250 \times 40$ & $250 \times 40$ \\
opt. surface size $\left(\mathrm{mm}^{2}\right)$ & $180 \times 20$ & $300 \times 20$ & $90 \times 16$ & $90 \times 16$ & $90 \times 16$ & $240 \times 25$ & $240 \times 20$ & $240 \times 20$ \\
rms roughness $(\mathrm{nm})^{*}$ & 0.31 & 0.27 & 0.2 & 0.14 & 0.15 & 0.24 & 0.29 & 0.25 \\
rms slope error tan. $(\mu \mathrm{rad})^{*}$ & 1.1 & 0.5 & 0.5 & 0.5 & 0.34 & 2.5 & 2.5 & 3.9 \\
rms slope error sag. $(\mu \mathrm{rad})^{*}$ & 10.4 & 0.8 & 1.25 & 0.8 & 0.9 & 5 & 25 & 25.5 \\
bulk material & $\mathrm{Cu}$ & $\mathrm{Cu}{ }^{\dagger}$ & $\mathrm{Si}$ & $\mathrm{Si}$ & $\mathrm{Si}$ & $\mathrm{Si}$ & quartz & quartz \\
coating (thickness: $30 \mathrm{~nm})$ & $\mathrm{Pt}$ & $\mathrm{Pt}$ & $\mathrm{Au}$ & $\mathrm{Au}$ & $\mathrm{Au}$ & $\mathrm{Pt}$ & $\mathrm{Pt}$ & $\mathrm{Pt}$ \\
source distance $(\mathrm{m})$ & 18.5 & - & $\infty$ & $\infty$ & $\infty$ & $22 / \infty$ & 2.5 & 1.75 \\
image distance $(\mathrm{m})$ & $\infty$ & - & $\infty$ & $\infty$ & $\infty$ & $5 / 6$ & 1.5 & 2.25 \\
total deflection angle $\left({ }^{\circ}\right)$ & 176 & $155-180$ & $155-180$ & $155-180$ & $155-180$ & 178 & 178 & 178 \\
long radius $(\mathrm{mm})^{*}$ & - & - & - & - & - & 462000 & 107450 & 112770 \\
short radius $(\mathrm{mm})^{*}$ & 1301 & - & - & - & - & 209.3 & 26.165 & 34.34 \\
line density $(1 / \mathrm{mm})$ & - & - & 300 & 1200 & 600 & - & - & -
\end{tabular}

\footnotetext{
* measured values

$\dagger$ GlidCop with electroless nickel plating as polishing layer (thickness: $\approx 100 \mu \mathrm{m}$ )

${ }^{* *}$ blaze angle $\left({ }^{\circ}\right)$
}

\section{Optical Layout, Performance Measurements and Polarization Control}

The schematic layout of the beamline is shown in Fig. 1 and selected parameters of the optics are summarized in Table 1. The horizontally deflecting cylindrical mirror collimates the light from the undulators in the vertical direction. The monochromator is equipped with the plane mirror and three gratings, two laminar (300/mm, and 1200/mm) and a blazed one $(600 / \mathrm{mm})$. Downstream we have a toroidal mirror for astigmatic focusing, horizontally to a mechanical chopper and vertically to the exit slit. The first toroidal refocusing mirror images these intermediate foci to the PEEM. In sequence there is the second toroidal refocusing mirror which does approximately a 1:1 imaging for a user-brought station. The mirror is retractable to allow also experiments with lower photon density and a spot size: $(2 \times 4) \mathrm{mm}$ FWHM $(v \times h)$.

The radiated power from the undulators into the acceptance of the beamline can go up to about $1 \mathrm{~kW}$. We use GlidCop mirrors with internal water channels directly underneath the mirror surface ${ }^{2}$ to apply intensive cooling at the first optical elements.

Measurements of the photon flux as function of photon energy and $c_{f f}$ parameter are shown in Fig. 2. The 600/mm blazed grating can provide at maximum about 5 times the flux of the 1200/mm laminar grating. On the other hand the resolving power of the $1200 / \mathrm{mm}$ is better by a factor 1.4 for comparable $c_{f f}$ settings. The ultimate resolving power of the $1200 / \mathrm{mm}$ grating can be increased further by operating at higher $c_{f f}$. The measurement confirmed the expectation that the blazed grating provides the high flux only closed to the "on blaze" condition while the laminar grating provides a bigger flexibility to choose $c_{f f}$ without a significant compromise in photon flux.

Fig. 3 shows examples for the resolving power of the beamline. We measured photoion yield spectra with a gas cell after the exit slit ${ }^{3}$. The measured resolving power is well beyond 5000 which is suited for X-ray absorption spectroscopy. To estimate the resolving power of $\mathrm{N}_{2}$ we used the method proposed by Chen [4] and compare the ratio

\footnotetext{
1 The actual ratio is $1.75: 2.25$ due to space constraints.

${ }^{2}$ Some details: 7 channels, width: $1 \mathrm{~mm}$, depth: $6 \mathrm{~mm}, 1 \mathrm{~mm}$ gap, $1 \mathrm{~mm}$ hot wall thickness, guard vacuum, joints: DN16CF flanges which cover the connections to the silicone hoses with an inner $\oslash$ of $10 \mathrm{~mm}$, water flow rate: up to $10 \mathrm{l} / \mathrm{min}$.

3 The gas cell belongs to the beamline diagnostics and is permanently installed between exit slit and first refocusing mirror. $100 \mathrm{~nm}$ thick Al windows can be inserted on both sides to separate the gas from beamline vacuum.
} 

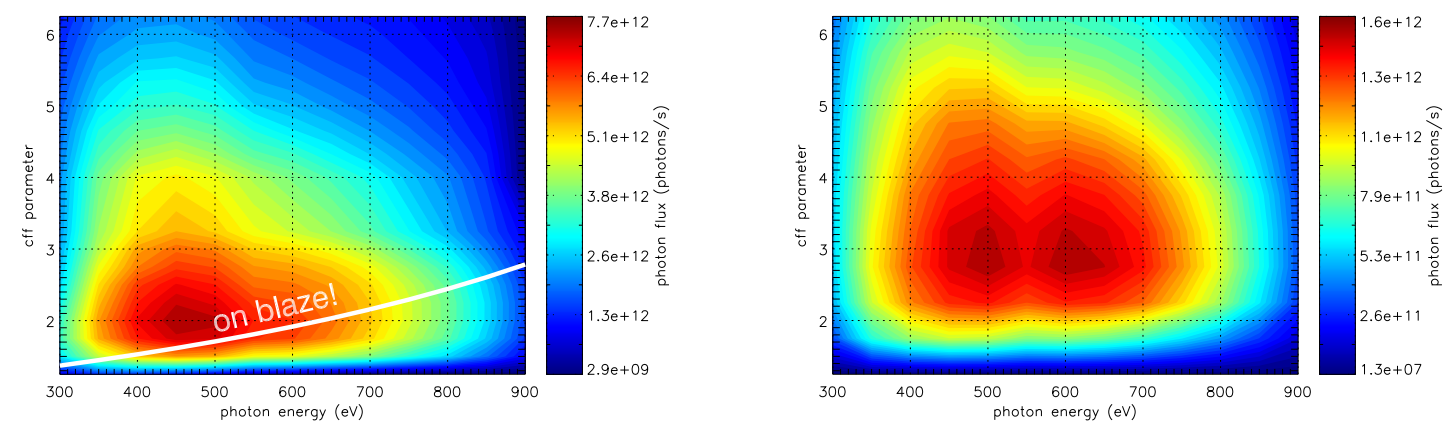

FIGURE 2. Measured photon flux as function of photon energy and $c_{f f}$ parameter. Blazed grating $600 / \mathrm{mm}$ left, "on blaze" condition is indicated. Laminar grating 1200/mm right. Parameter: ring current, top up $401 \mathrm{~mA} \pm 1 \mathrm{~mA}$, ID1 + ID2, no phase matching, linear horizontal polarization, first harmonic, acceptance: $(1.25 \mathrm{~mm})^{2}$ at $13.6 \mathrm{~m}$, exit slit: $20 \mu \mathrm{m}$, this corresponds for instance to a resolving power of 6200 for the $600 / \mathrm{mm}$ and 8700 for the $1200 / \mathrm{mm}$ at $700 \mathrm{eV}$ and $c_{f f}=2.25$. The local minimum around $550 \mathrm{eV}$ is related to a carbon/oxygen contamination at the optics.
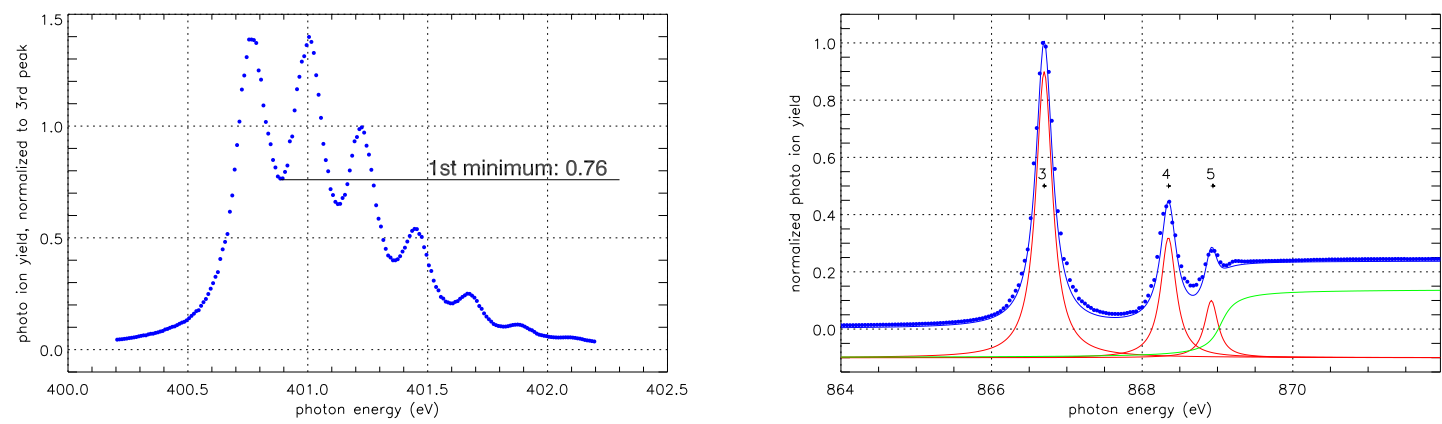

FIGURE 3. Photoion yield measurements with a gas cell to demonstrate the resolving power of the beamline. Nitrogen left and Neon right. Parameter: ID2 lin. hor., $1200 / \mathrm{mm}$ grating, $c_{f f}=5$, exit slit $\mathrm{N}_{2}: 20 \mu \mathrm{m}$, Ne: $10 \mu \mathrm{m}$.

of the first minimum and third maximum. We determined 0.76 and compared this with simulated Voigt profiles where we assumed a Lorentzian width of $112 \mathrm{meV}$ which is the lower limit of the diverging data found in literature. With such a conservative assumption the resolving power is between 5000 to 8000 . The neon spectra has been fitted with 3 Lorentzians and an arcus tangens background. For the Lorentzian width we use recent data from [5], for the first peak: $\mathrm{FWHM}=0.132 \mathrm{eV}$. The fit represents the measured data already very good- we state the resolving power is better than $6500 \approx 866 \mathrm{eV} / 0.137 \mathrm{eV}$ where $0.137 \mathrm{eV}$ is the FWHM width of the first peak.

The size of the focus has been measured with a phosphor screen and CCD camera after the second refocusing station. We determined $(45 \times 60) \mu \mathrm{m}(v \times h)$, Fig. 4 left. Comparable results have been received by scanning the PEEM for the first focus (Fig. 4 right).

The beamline offers full control of the polarization with a fast switching capability. This is essential in particular for magnetic imaging with different polarizations. The time to switch should be short in order to minimize possible drifts between two images. To accomplish this routinely we use two Apple II type undulators ${ }^{4}$ in sequence and take advantage of the small energy bandpass of the monochromator (width $\approx$ meV). The undulators deliver the X-rays in harmonics with a specific, finite energy width in the range of a few $\mathrm{eV}$. The central energy of the harmonics depends on the gap and shift settings. To deliver X-rays to the experiments, the energy of the harmonics has to fit to the

\footnotetext{
${ }^{4}$ Some undulator parameters: linear horizontal polarization from $90 \mathrm{eV}$ to $2 \mathrm{keV}$, optimum range: first harmonic: $90-750 \mathrm{eV}$, third harmonic: $600-$ $1600 \mathrm{eV}$, fifth harmonic: 1- $2 \mathrm{keV}$, circular left/right polarization: from $140 \mathrm{eV}$ to $950 \mathrm{eV}$ in first harmonic, degree of polarization: $>97 \%$ (confirmed by measurements with the BESSY diffractometer [6]), elliptical polarization with third harmonic up to $2 \mathrm{keV}$, linear vertical polarization: $180 \mathrm{eV}$ to $2 \mathrm{keV}$.
} 

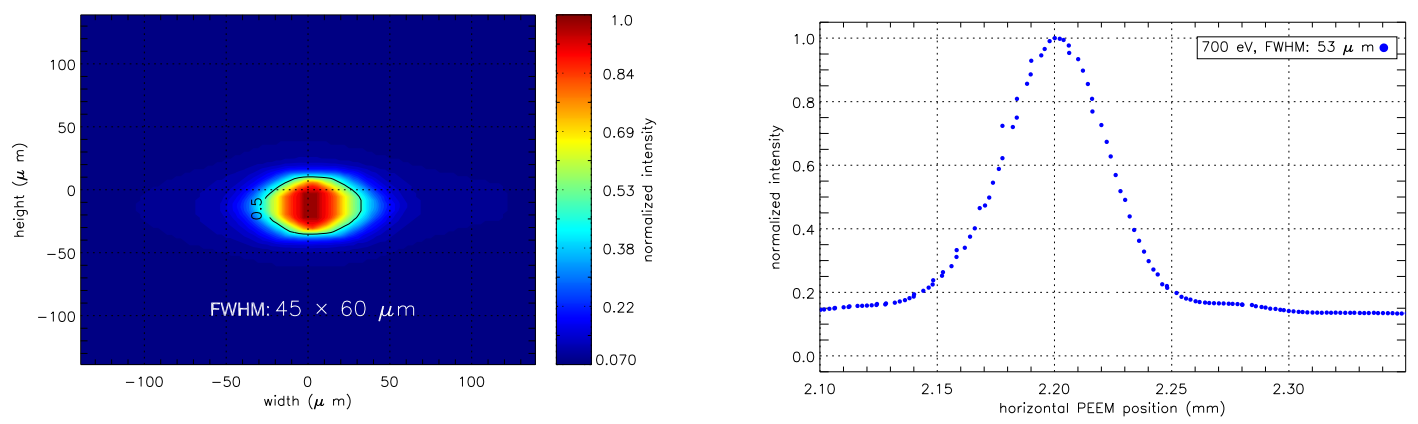

FIGURE 4. Spot size measurement. The left figure shows the normalized intensity at a phosphor screen in the focus of the second refocusing mirror imaged with a CCD camera. The measured FWHM is indicated by the solid line in the contour plot. Parameter: ID2 lin. hor., $1200 / \mathrm{mm}$ grating, $800 \mathrm{eV}, c_{f f}=2.25$, exit slit: $30 \mu \mathrm{m}$. The right figure shows horizontal spot size measurements by scanning the PEEM position with comparable parameters.
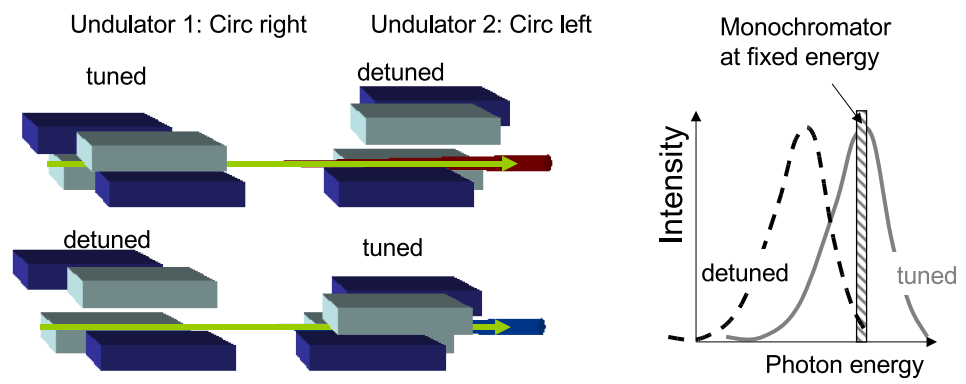

FIGURE 5. Polarization switching scheme. Detuning one of the undulators shifts it's intensity to lower photon energies- out of the energy acceptance of the monochromator which is matched to the intensity provided by the other undulator.

monochromator setting i.e. they have to be tuned. Typically we set one undulator to circular left, the other one to circular right polarization. Detuning one undulator by changing now the gap or shift parameter by a few millimeters will shift the center of the emitted photon energy away from the energy bandpass of the monochromator ${ }^{5}$ such that nearly no intensity from the detuned undulator but the full intensity from the tuned one passes the monochromator. The scheme is depicted in Fig. 5. Since the required mechanical movements are small the polarization switching can be performed within a few seconds. The originally planned scheme with parallel displaced beams in the undulators and a mechanical chopper at the intermediate focus [7] has been abandoned ${ }^{6}$.

\section{REFERENCES}

1. R. Follath, and F. Senf, Nucl. Instr. Meth. Phys. Res. A 390, 388-394 (1997).

2. U. Flechsig, L. Patthey, and C. Quitmann, Nucl. Instr. Meth. Phys. Res. A 467-468, 479-481 (2001).

3. U. Flechsig, et al., "Performance Measurements at the SLS," in SRI 8, 2004, vol. 705 of AIP Conf. Proc., pp. 316-319.

4. C. T. Chen, and F. Sette, Rev. Sci. Instrum. 60, 1616-1621 (1989).

5. M. Kato, et al., "Absolute Photoionization Cross Section," in SRI 9, 2007, vol. 879 of AIP Conf. Proc., p. 1121.

6. F. Schäfers, H.-C. Mertins, A. Gaupp, et al., Appl. Opt. 38, 4074-4088 (1999).

7. C. Quitmann, et al., Surf. Sci. 480, 173-179 (2001).

\footnotetext{
${ }^{5}$ It is preferable to shift the detuned harmonics towards lower photon energies to take advantage of asymmetry of the harmonics.

${ }^{6}$ Reasons: a) The storage ring and beamline works so stable and reproducible that the benefit of fast switching for noise reduction (lock-in technique) was negligible (we reached $20 \mathrm{~Hz}$ ). b) The calibration due to the different beam paths down to the $10^{-4}$ level is very difficult. c) To overlap the beams, the microscope has to be moved along the beam path by several $\mathrm{cm}$ and back for focused operation. This is problematic with such a heavy station like the PEEM.
} 